\title{
Metropolitan age-specific mortality trends at borough and neighbourhood level: The case of Mexico City
}

\section{Karol Baca-Lopez}

Instituto Nacional de Medicina Genomica

Cristobal Fresno

Instituto Nacional de Medicina Legal e Ciencias Forenses

Jesus Espinal-Enriquez

Instituto Nacional de Medicina Genomica

Miriam V. Flores-Merino

Universidad Autonoma del Estado de Mexico

Miguel A. Camacho-Lopez

Universidad Autonoma del Estado de Mexico

Enrique Hernandez-Lemus ( $\nabla$ ehernandez@inmegen.gob.mx )

Instituto Nacional de Medicina Genomica https://orcid.org/0000-0002-1872-1397

Research

Keywords: All-cause mortality, Spatio-temporal statistics, Ordinary Kriging

Posted Date: January 2nd, 2020

DOI: https://doi.org/10.21203/rs.2.19611/v1

License: (9) (i) This work is licensed under a Creative Commons Attribution 4.0 International License.

Read Full License 


\section{RESEARCH}

\section{Metropolitan age-specific mortality trends at borough and neighbourhood level: The case of Mexico City}

Karol Baca-López ${ }^{1,2 \dagger}$, Cristóbal Fresno ${ }^{3 \dagger}$, Jesús Espinal-Enríquez ${ }^{2}$, Miriam V Flores-Merino ${ }^{4}$, Miguel A Camacho-López ${ }^{1}$ and Enrique Hernández-Lemus ${ }^{2,5^{*}}$

\footnotetext{
${ }^{*}$ Correspondence:

ehernandez@inmegen.gob.mx ${ }^{2}$ Computational Genomics

Department, National Institute of Genomic Medicine, 14610 Mexico City, Mexico

Full list of author information is available at the end of the article

${ }^{\dagger}$ Equal contributor
}

\begin{abstract}
Background: Understanding the spatial and temporal patterns of mortality rates in a highly inhomogeneous metropolis, is a matter of public policy interest. In this context, there is no previous study that correlates both spatio-temporal and age-specific mortality rates in Mexico City.

Methods: Spatio-temporal kriging modelling was used over six age-specific mortality rates (from the years 2000 to 2016 in Mexico City), to gain both spatial (borough and neighbourhood) and temporal (year and trimester) data level description. Resulting data were modelled using time-evolution mixed effect models to unblurred mortality age-specific patterns. Posterior tests were carried out to compare mortality averages between geospatial locations.

Results: Mortality correlation extends in all study groups for as long as 12 years and as far as $13.27 \mathrm{~km}$. The highest mortality rate takes place in the Cuauhtémoc borough, as it is the commercial, touristic and cultural core downtown Mexico City. On the contrary, Tlalpan borough is the one with the lowest mortality rates in all the study groups. Interestingly, post-productive mortality is the first age-specific cause of death, followed by infant, productive, pre-school and scholar groups.
\end{abstract}

Conclusion: The combinations of spatio-temporal Kriging estimation and time-evolution mixed effect models, allowed us to unveil relevant time and location trends that may be useful for public policy planning in Mexico City.

Keywords: All-cause mortality; Spatio-temporal statistics; Ordinary Kriging

\section{Introduction}

Identifying trends in Metropolitan Mortality (MM) is one of the most challenging problems nowadays. Systematic approaches to discriminate the relevance of social, economical, demographic, educational, environmental or criminal factors in MM are matters of intense current research $[1,2,3]$. The MM can be differentiated from rural mortality given the fact that causes and risk factors are substantially different, even when comparing their rates [3]. To develop a useful model that could explain the evolution in time and space of MM, it is crucial to have a comprehensive dataset with information regarding the aforementioned factors. However, missing data is one of the most common troubles in developing countries, particularly when talking about small-scale spatio-temporal level measurements, such as, neighbourhood and monthly/seasonal respectively. 
One of the most useful data to explain MM is the age group, since the causes associated to risk factors, disease incidence and other several related variables, are substantially different between these groups. Seminal works regarding age-specific MM can be found elsewhere $[4,5,6]$. Often, these age groups are separated into nonoverlapping sets as follows: i) Infant, ii) Pre-school, iii) School, iv) Productive and v) Post-productive. On the other hand, mortality as well as other health outcomes, can equally be described at different scales, for example, individually and collectively $[7,8]$. It is well known that individual mortality is influenced by personal-level characteristics such as genetics, socioeconomic status and education. However, there is growing evidence that collective or regional disadvantages can also be good predictors of individual mortality and population level mortality $[9,1]$. Example of these regional characteristics or more broadly, regional disparities, can be classified into social, demographic and environmental factors. In a recent study, Gavourova and Toth (2019) described how environmental factors influence changes in preventable mortality and how they impact differ from district to district in Slovakia [2]. In another context, individual and regional characteristics have been simultaneously analyzed for cardiovascular disease, to elucidate the effects of their interaction with air pollution, psychosocial stress, adverse childhood experiences and neighborhood deprivation index [10].

In regard to the description of spatial mortality trends, there exists basically two main opposite approaches: i) All-cause or ii) Cause-specific mortality studies. In the first case, all-cause mortality rates, including its spatial and temporal variability have been measured, using different levels of granularity [11, 12, 13, 14, 15]. Contrarily, several cause-specific mortality models have been developed for diseases such as cancer, diabetes, hypertension, chronic obstructive pulmonary disease, cardiovascular disease, hepatitis C and HIV/AIDS [16, 12, 15, 17, 18, 19, 20, 21, 22, 6, 23, 24]. As an example of this approach, Dwyer-Lindgren et al. (2017), studied the variations in life expectancy, mortality rates and years of life lost from 152 causes of death at the county and neighbourhood levels by age group and sex. They mainly conclude that county level estimations mask important local differences (between neighbourhoods) [13, 25]. Studies with these characteristics have been performed for developed countries which generally have big, accessible and almost complete databases. In the case of developing countries, it has been described an exacerbated problem in health disparities due to poverty, environmental threats, inadequate access to health care and educational inequalities that could lead to higher mortality rates [4]. Despite these studies rely on partially documented health and socio-demographic registries, it has been possible to gain insights of spatially differences in health outcomes.

In order to have a more robust input dataset to analyze trends in MM, the measured variables must have a temporal component as fine-grained as possible, since it is well known that several risk factors associated with mortality have a cyclic behavior, such as temperature, air pollution, seasonal pathogens or even individual social aspects $[26,27,28]$. Several studies on MM have taken into account yearly data to associate certain variables with the response outcome $[18,19,9,20]$. Often, monthly or trimester data are not available in developing countries. In other cases, there is a great number of missing data in several fields. In general, although different area-level health outcomes might share a variety of explanatory variables 
such as socioeconomic, pollution, delinquency levels, health access among others; by down scaling, spatio-temporal heterogeneity might arise [29, 18, 19, 30, 31]. To overcome this problem, a variety of geo-statistical procedures have been implemented to estimate mortality rates at different granularity levels. For instance, PopulationWeighted Average, local and global Empirical Bayes and Poisson Kriging have been used to estimate disease-specific mortality rates from age-adjusted data. Accounting for spatial correlation patterns for low and high frequency rates, Poisson Kriging have shown better results [32, 33]. Kriging methods allow to estimate spatial risk considering heterogeneity among small areas from poorly reported databases [34]. To overcome data scarcity, Kriging approach has been used to interpolate spatial missing data at the county level as well as for temporal interpolation in mortality data $[35,6,11]$. Kriging estimates has been also used to describe changes in child mortality trends, evaluating between-countries and within-country sources of variation [5]. However, despite of these studies, there is no consensus about which spatial or spatio-temporal methodology is the best for this purpose.

Mexico City, is one of the most populated cities worldwide. Although its economy, employment rates and health services have improved over the last decades. Unfortunately, they are unevenly distributed mainly affecting three risk groups: the children, the elderly and the poor [36]. To worsen this situation, exposure to environmental risk factors derived from urbanization and migration, have increased with their associated negative health effects which are gaining attention in recent years.

In a recent study, Aburto et al. (2018) analyzed lifespan and preventable mortality for the 32 states in Mexico, including Mexico City. They described changes for age groups and particularly reported an increase in Diabetes mortality rate [37]. However, at borough and smaller scales; health, socio-economic, educational and environmental disparities for Mexico City have not been formally quantified. In general, quality of life indexes of the urban and rural blocks are drastically different, but, more importantly, between urban neighbourhoods these differences may be larger than between rural and urban blocks. Recently, new estimations have suggested a high degree of social backwardness [38]. In Mexico City, health disparities may be enormous between close neighbourhoods, even if they belong to the same borough. Taking this into account, to evaluate health outcomes such as MM using borough as a measurement unit, could result in an inaccurate assessment. A similar behaviour arise if we consider the temporal component: coarse-grained measurements may mask the variant behaviour of MM. Despite it is well known that seasonal variations in environmental factors, as well as socio-economical factors may influence interpretation of the available data for Mexico City, there is no previous report in which time lapses for measurements were taken in smaller scales than in a year time. Nevertheless, both spatio-temporal data are available in Mexico City for MM. These data include, at the borough level, information for general mortality, age, gender and other descriptive variables from 2000 to 2016 in a year basis.

In this work, using available data for Mexico City, a two-level both spatial and temporal description for MM was analysed using geo-statistical interpolation. In this context, the spatial component is tackled at borough and neighbourhood scale, whereas the time scale at a year and trimesters. Thus, a finer description level of 
this phenomena could result in a more accurate spatio-temporal description of the MM in Mexico City.

\section{Methods}

\section{Study area}

The study area is the capital of Mexico, Mexico City. The geo-spatial information, i.e., polygon shapefiles, were obtained from the citizen open data initiative website at Datamx.io [39]. The city is divided into 16 administrative boroughs (municipalities) and 2,097 neighbourhoods, according to the 2015 data description. The neighbourhood polygons were combined by borough identifiers to create the corresponding borough regions (Fig. 1A), in order to have a two-level hierarchical description of Mexico city. Borough centroids, were obtained using rgeos $\mathrm{R}$ package [40] (Figure 1B). In addition, neighbourhood centroids were obtained by gCentroid or gPointOnSurface for convex or concave polygons respectively (data not shown).

\section{Mortality database}

A mortality database was created from available open data obtained at the Secretariat of Health in Mexico City (Secretaría de Salud de la Ciudad de México: SEDESA) [41]. The database comprehends a 16 borough, age-specific mortality rates follow up complete records, from the year 2000 up to 2016 and can be downloaded from [42]. The age-specific groups include the following five non-overlapping age descriptors: i) Infant ( $x<1$ years old), ii) Pre-school $(1 \leq x<4$ years old), iii) School ( $4 \leq x<14$ years old), iv) Productive $(14 \leq x<64$ years old) and v) Post-productive ( $x \geq 64$ years old). In addition, the global mortality rate group was considered, independent of the age-specific descriptor. The mortality rates are expressed, in all cases, per 1,000 inhabitants, but the Infant rate, which was calculated per 1,000 live-born according to the data obtained from the Mexican National Institute of Statistics and Geography (Instituto Nacional de Estadística y Geografía, INEGI) and the Mexican National Population Council (Consejo Nacional de Población, CONAPO) [43, 42].

A five year time-step borough level evolution descriptive panel for every agespecific group is presented in Figure 2. The rows stand for age-specific mortality rate, whereas, columns for a summarized time period (year) as reported by the SEDESA. With the available data at hand, the only alternative to visually explore the spatial data component, is to homogeneously colour each borough with a single mortality rate for the reported year. Fortunately, there is continuity among neighboring boroughs, i.e., there are no big transitions between adjacent boroughs. In this representation the time evolution is not captured as well as the spatial mortality age-specific rates. To overcome this drawback, a visual description at borough level is presented in Figure 3.

In Figure 3 can be seen that there are different age-specific time evolution patterns, such as decreasing mortality rates for Infant and Post-productive groups, in contrast to the increasing trend for Global and Productive cases. Although the Cuauhtémoc borough (in green) is the smallest one in terms of its neighbourhoods (38 in total), it seems to overcome the mortality rates for most age-specific cases in comparison to the rest of the boroughs. On the other hand, no apparent time dependance correlation can be describe for School and Pre-school, where several borough vibrant mortality curves are presented. 


\section{Spatio-temporal interpolation}

The spatio-temporal description of the mortality database used in this work is on a yearly basis at borough level. Although, both spatial and temporal description levels can be accurate for open data summarized statistics, it fails to represent the actual statistical metric unit, e.g., trimester measurements at neighbourhood level. In addition, it is known that in this context, data points have a spatio-temporal correlation which can be used to estimate values at unmeasured locations in space and time. Among the different geo-statistical interpolation methodological alternatives, the Kriging family provide unbiased estimates that minimize the variance-covariance spatio-temporal structure using an appropriate variogram modelling [44].

Briefly, a second data description level in both space and time, using neighbourhood centroid locations and a trimester time basis, was carried out using Ordinary Kriging, for each age-specific mortality rate. All estimations were obtained using gstat $\mathrm{R}$ package implementation [45, 46]. Initial variogram parameter values were obtained from the complete mortality empirical (s)patio-(t)emporal rates variogram matrix $\gamma(s, t)$ (Supplementary Table 1$)$ :

- Nugget: The median value of the first three empirical variogram matrix row/column means, for the spatial or temporal initial guess respectively.

- Range: The spatial range is one third of the lagged maximum spatial value and for the temporal case, it corresponds to the maximum value.

- Sill: The median value of the last five empirical variogram matrix row/column means, for the spatial or temporal initial guess respectively.

- stAni: The spatio-temporal anisotropy was estimated using a linear model as implemented in gstat $\mathrm{R}$ package $[45,46]$.

- Joint spatio-temporal initial values, are based on the mean of the independent spatio- and temporal values respectively.

Using the initial variogram parameters, different spatial, temporal or joint covariance structures were tested to find the best parsimonious correlated data description, according to the available implementations in gstat (metric, separable, productSum, sumMetric and simpleSumMetric) [45, 46]. All possible single, double or triple variogram combinations (Exponential, Gaussian and Spherical) were tested according to the corresponding covariance structure. All covariance models were fitted using a quasi-Newton box constrained method, where only the lowerbound was set to 0.001 for every parameter. The upper-bound was left to its default value (infinitum), i. e., no box constraint was imposed for the maximum value. The covariance structure model selection criterion used was to minimize the weighted mean squared error. Complete age-specific mortality tested covariance fitted model results can be found in Supplementary Table 2. Finally, the spatio-temporal mortality rate interpolation was obtained using all the available data points, under the best covariance model description, for the neighbourhoods centroids at a trimester level, in the period 2000 to 2016.

\section{Time-evolution modelling}

Age-specific mortality rates time evolution was modelled using a Mixed-effects Linear Model to account for data constraints and lack of error independence using the definitions of Equations (1-4) [47]. The model was specified using Infostat software version 2018 as follows [48]: 


$$
\begin{array}{r}
y_{i j k}=\mu+\alpha_{i}+\beta_{j}+\gamma \times t_{k}+\delta \times t_{k}^{2}+\zeta \times t_{k}^{3}+\alpha_{i} \times \beta_{j}+\alpha \times \gamma \times t_{k}+ \\
\alpha_{i} \times \delta \times t_{k}^{2}+\alpha_{i} \times \zeta \times t_{k}^{3}+\beta_{j} \times \gamma \times t_{k}+\beta_{j} \times \delta \times t_{k}^{2}+\beta_{j} \times \zeta \times t_{k}^{3}+ \\
\alpha_{i} \times \beta_{j} \times \gamma \times t_{k}+\alpha_{i} \times \beta_{j} \times \delta \times t_{k}^{2}+ \\
\alpha_{i} \times \beta_{j} \times \zeta \times t_{k}^{3}+\varepsilon_{i j k} \\
\varepsilon_{i j k}=\lambda_{t_{k}}+\nu_{i j k} \\
\lambda_{t_{k}}=\phi_{\beta_{j}} \lambda_{t_{k-1}}+u_{t_{k}} \\
\operatorname{var}\left(\epsilon_{i j k}\right)=\sigma^{2} g^{2}\left(\alpha_{i}\right)
\end{array}
$$

where, $y_{i j k}$ is the mortality rate for the $i$-th age-specific mortality group $\left(\alpha_{i}\right)$, at the $j$-th borough level $\left(\beta_{j}\right)$, for the $k$-th time $\left(t_{k}\right) ; \mu$ is the global mean; $\gamma, \delta, \zeta$ are the corresponding third order time polynomial coefficients; in addition, the double and triple complete fixed effect model interactions and the error term $\varepsilon_{i j k}$ were also specified. Indeed, the error term $\varepsilon_{i j k}$ in Equation (1) is modelled in Equation (2) by a two-level model, to account for the lack of independent errors; where, $\lambda_{t_{k}}$ denotes the unobservable time effect and $\nu_{i j k}$ is the idiosyncratic error term. The correlated errors were tackled using a first order auto-regressive model for time dependence as described by Equation (3), where $\phi_{\beta_{j}}$ is the corresponding coefficient and $u_{t_{k}}$ the individual effect. Finally, the heteroscedasticity in Equation (4) was modelled as a multiplicative effect of the residual variance $\sigma^{2}$ times the variance error function $g($.$) using a varIdent definition for the different age-specific mortality groups \alpha_{i}$ [48].

The model was fitted using $\mathrm{R}$ language with the nlme package under restricted estimation of the maximum likelihood $[49,50]$. When possible, back-step model selection strategy was applied to remove the least significant fixed-effect term, one at the time, until no difference was found using a maximum likelihood test between competitors models. Type III sum of squares was used to assess an Analysis of Variance (ANOVA) table for marginal hypothesis tests for the fixed effects. Posterior Fisher's Least Significance Difference (LSD) tests were applied over statistical significant terms, using a multiple comparison Bonferroni p-value correction. When possible, bilateral test was used and the Type I error was set to 0.05.

\section{Results}

\section{Spatio-temporal variogram estimation}

Regarding the spatio-temporal age-specific mortality estimations, the initial guesses obtained from the sample spatio-temporal variograms are shown in Supplementary Table 1 (see material and methods section). Depending on the age-specific mortality group, the initial guesses are different for the nugget, range, sill and spatio-temporal anisotropy (stAni). In this context, the nugget is the model intercept attributable to measurement errors or spatial sources of variation at distances smaller than the sampling interval or both. Interestingly, these sources of variations are negligible for Pre-school and School, in contrast to the other mortalities, with a wide range of nugget values $(0.01-21.31)$. In addition, the correlation extends between measurements, also known as range, in all cases is exactly the same for all mortalities and 
last about 12 years for as far as $13.27 \mathrm{~km}$. The variogram values obtained in the range, a.k.a the sill, is as close to the nugget for the school and pre-school mortalities and departs from it at most to double its value for Infant. The anisotropy remains the same for global, post-productive, school and infant, but differ for productive and pre-school.

Final covariance model weighted mean square error for all the tested variogram permutations can be found in Supplementary Table 2. It is worth to mention that the lowest error for the different covariance structure methods included the metric for Global, School and Pre-school; sumMetric for Productive and Infant and simpleSumMetric for Post-productive was the best one. Within these covariance models, there was no apparent pattern in the winner variogram model permutation (temporal + spatial + joint $)$. The Gaussian + Gaussian $(\mathrm{Gau}+\mathrm{Gau})$ was the winner's choice for temporal and spatial in Post-productive. Moreover, Infant mortality also followed this pattern, with an additional Gau component for the joint variogram. The Exponential + Gaussian (Exp + Gau) was the winner choice for Productive mortality and Gau joint variogram. Indeed, this is a data-driven approach that required to explore the complete permutation grid, in order to reach a parsimonious spatio-temporal correlation model. A visual comparison of each winner covariance model and sample variogram can be found in Supplementary Figures 1-6.

\section{Spatial two-level mortality rate description in Mexico City}

The spatio-temporal mortality description in Mexico City starts at the raw data presented in Figure 2. Now, let's consider the complete picture, using, for example, global mortality rate as presented in Figure 4. Using the borough spatio-temporal granularity (Figure 4A), there is a clear spatial pattern, no matter the selected year $(2000,2005,2010$ or 2015). This pattern has a global maximum mortality rate value (hotspot) at Cuauhtémoc borough (number six in Figure 1A). There, the mortality rate values radially decrease as long as we depart from this location to the outer boundaries of Mexico city. Indeed, the decrease is not homogeneous, i. e., is dictated by a spatial anisotropy where the north and northeast direction have a less marked decline in comparison to the south and south-west direction. Moreover, at this temporal description, there is also an increment in the global maximum rates as we move from 2000 to 2015, in concordance with the temporal patterns presented in Figure 2 for the Global case.

Moving towards a deeper data exploration, the spatio-temporal interpolation obtained by Ordinary Kriging provides a Global mortality rate smooth surface (Figure 4B). For a fair comparison, the same time scale was used (years), whereas the spatial description considered the neighbourhood centroids grid. In this spatially zooming, it can be seen a more realistic geographical-continuous mortality transitions, unlike the discrete phenomena at borough level for next door boroughs presented in Figure 4A. In other words, this reflects a far less abrupt change from one borough to another, thus giving a continuity between neighbourhoods. Moreover, it remains consistent between both spatial-scales, that from the year 2000 until 2015 the Global mortality rates increase throughout neighbourhoods and boroughs.

In the last panel, Figure 4C, an individual borough representation is displayed to distinguish neighborhood level trends. Here, Cuauhtémoc borough was selected for: 
i) Having most of the times the highest values for Global, Post-productive, Productive and Infant mortality rates throughout all boroughs and years (see Figure 3) and ii) Visualization purposes as it is composed by 38 neighbourhoods, the smallest borough as reported in 2015 [39]. Although apparently imperceptible, there are distinguishable differences in mortality values among Cuauhtémoc neighbourhoods. Similar differences are obtained for the rest of the boroughs (data not shown).

\section{Time-evolution mortality rate modelling in Mexico City}

The borough-level age-specific mortality rate data were modelled using Equations (1-4). Interestingly, the ANOVA results presented in Table 1 show that the only not significant effect at borough level $(p=0.22)$, is the triple interaction that includes the time to the third power. Hence, the mortality rate time-evolution pattern in Figure 3 has a complexity that can be parsimoniously captured by our methodological proposal. In addition, the autocorrelation parameter had an impact not as high as one would expect at borough level $(\phi=0.12)$. On the other hand, the variance function did address the different age-specific groups where Infant was the one with the highest value (11.59) followed by Post-productive (10.34), Global (1.00), Pre-school (0.85), Productive (0.82) and School (0.43).

To further explore the mortality behaviour, posterior Fisher's LSD tests were obtained over the age-specific, borough and age-specific times borough double interaction terms (Figure 5). It is worth to notice, that the age-specific mortality rate groups do not overlap between each other, due to the different Fisher's LSD letters (A-F) in Figure 5A. Moreover, the Post-productive group (A) outruns any other group, but also doubles its following competitor, the Infant group (B). Interestingly, this trend also remains when we compare every two consecutive groups, i.e., B vs. $\mathrm{C}, \mathrm{C}$ vs $\mathrm{D}$ and so on. In this age-specific context, the school is the group (F) with the least mortality rate. On the other hand, the mortality rate at the 16 different boroughs (Figure 5B) showed Fisher's LSD group letters from A to I, where the Cuauhtémoc (A) and Tlalpan (I) are the boroughs with the highest and lowest model estimated mean values respectively. Now, if we assess the posterior results over the age-specific and borough double interaction term (Figure 5C), they suggest that the age-specific LSD groups pattern in Figure 5A is now discriminated among boroughs, almost following the evidence presented in Figure 5B.

If we turn our attention at neighbourhood-level for the borough with the highest mortality rate, i. e., Cuauhtémoc, we found that the model presented in Equations (1-4) is not well-suited for this level description data. Hence, a model selection process was considered in order to gain the best parsimonious data description. The current model results are presented in Table 1, where it can be seen that some cells are empty due to terms discarded from the analysis. Interestingly, the third and second order time triple interaction terms have been excluded from the analysis, in addition to the borough times time to the third power. Hence, in this context the modelling complexity has been reduced, at the expense of a higher autocorrelation $(\phi=0.88)$ and different variance function parameters and ranking, i. e., Productive (3.80), Post-productive (2.43), Pre-school (2.22), School (1.35), Global (1.00) and Infant (0.04). 
The Cuauhtémoc borough results can be found in Figure 6. This borough contains 38 neighbourhoods which were numbered according to the high mortality rate downwards (Figure 6A). This result is complemented by Figure 6B, where the Fisher's LSD letters were used to describe the landscape. Interestingly, at neighbourhood level, there is also a radial mortality rate decay starting at the central neighbourhood with number one and the letter A. The two neighbourhoods with the lowest mortality rate (letter D) are situated at the opposite borough extremes - south-west ant northeast borders. Moreover, the age-specific mortality patterns have changed from Post-productive, Infant and Global mortality at the borough description in Figure 5 into Global, Post-productive and Pre-school as seen in Figure 6C. Finally, the Fisher's LSD test results in this borough, but at neighbourhood level, showed some regions with up to four possible over-lapping letters. It is worth noticing that in the opposite extremes there is Tabacalera (A) and in the other end is shared by Valle Gómez and Hipódromo de la Condesa.

\section{Conclusions}

Understanding MM is not only relevant in terms of a mere descriptive statistical approach but, to take into account variables that could determine, with the highest accuracy, this crucial health outcome. This knowledge, in due time, could lead to the establishment of appropriate public policies to improve citizen life quality in metropolitan areas. In the aforementioned terms, by means of the development of a modelling approach based on a systematic interpolation of missing data, one may observe spatio-temporal dynamics of mortality in the urban areas with higher precision. In this context, Kriging family methods have proven to be useful to achieve this goal. In this work we have demonstrated how the improvement of granularity level at both spatial and temporal definition, could explain some of the socio-demographic variables underlying the changes in mortality rates between boroughs. This task was achieved by statistically interpolating those data points into a fine-grained level. In what follows, we will comment on the findings using spatio-temporal Kriging-based methods.

It can be easily noticed that Cuauhtémoc borough is the one with the highest mortality rate in all age groups, but for pre-school -which ranks in third placeas depicted in Figure 3. This borough has several particularities that should be commented, in order to unblur some hints towards plausible explanations, for the unique behaviour observed there along time and space. As observed in Figure 5C, Cuauhtémoc is the borough with the most different mean value in all age groups, but in Pre-school, which shares places with Cuajimalpa and Iztapalapa boroughs. Except in that case, in the other four age-specific groups, Cuauhtémoc has an outstanding mortality behaviour over time. The case is particularly dramatic in the productive age (14 to 64 years old). In addition, in the Productive time-evolution pattern in Figure 3 can be observed the different temporal behaviour of Cuauhtémoc (solid green dashed line), compared to the rest of the boroughs. For one thing, Cuauhtémoc is the economic center of Mexico City. There, it is concentrated the executive and legislative powers, as well as the most important commerce hotspots. Indeed, Cuauhtémoc is the most densely populated borough of Mexico City. In economic terms, it concentrates $4.6 \%$ of the gross domestic product of the entire 
country [51]. A relevant point to take into account in Cuauhtémoc, is the fact that around 5 million people pass through this borough every day, despite its population oscillates only around 500,000 inhabitants. This is the place in Mexico City with the highest number of public transport stations. Cuauhtémoc also concentrates the largest markets of informal commerce of the city (Tepito Market, in the Morelos neighbourhood). The high density and the flux of money and services may help to explain, to some extent, the different behaviour of the mortality rate in the productive age in this borough.

By taking into account the spatio-temporal Kriging, it has been possible to modelled mortality rates at the neighbourhood level. With this model, the four places with the highest mortality rates were Tabacalera, Centro, Juárez and Doctores neighbourhoods. According to reports of the Executive Secretariat of the National System of Public Security System (Secretariado Ejecutivo del Sistema Nacional de Seguridad Pública, SESNSP), Centro is one of the most insecure boroughs of Mexico City and the homicide rate is the highest in the city [52]. By integrating transit and other accident-related issues, with employment determinants and crime-associated mortality, it may be possible to present an explanation for which the productive age mortality presents a consistent increase in Cuauhtémoc, compared to the rest of the boroughs.

The model presented here also has caveats, since it shows an interpolation of the coarse-grained data at the borough level of description. For instance, according to the model, Tabacalera is the neighbourhood with the highest mortality rate, however, by looking at the data, the homicide rate as an example is not as large as Centro or Morelos [52]. At this stage is not possible to disambiguate whether these inconsistencies are failures of the interpolating strategy or indeed reflect different causes of death, such as the ones related to environmental factors like air pollution and other contaminants. Despite this caveat, the model shows a radial decrease of mortality in Mexico City, starting from Cuauhtémoc downtown, similar to the general behaviour observed at the borough level (Figure 2).

The granularity enhancement in mortality and health outcomes data will further improve its usage in a variety of public policies, such as, urban development, security, creation of health centres, hospitals, public transport and even water re-usage. In these terms, efforts in gathering data at the lowest possible source (time and space), are highly desirable for future works. Finally, in order to build predictive models that allow to tailor public policy design, the spatio-temporal dynamics of MM should incorporate socio-demographic, environmental, economical factors and relevant covariates, as detailed as possible. Future directions include, but are not limited to, utilizing environmental data, such as air pollution, quality of water, disposition of water supplies among others. Some of the later variables are actually available as open data resources. The ultimate understanding of MM by using the data presented here, as well as environmental and other risk factors, will help in the searching for the improvement of life quality of the metropolitan areas in Mexico City.

Declarations

Ethics approval and consent to participate Not applicable. 
Consent for publication

All authors have agreed on the contents and have given their consent for publication.

Availability of data and material

All related materials are either in the manuscript, in the supplementary materials or available from the authors upon request.

Competing interests

The authors declare that they have no competing interests.

Funding

Not applicable

Authors' contributions

$\mathrm{KBL}, \mathrm{CF}, \mathrm{JEE}$ and $\mathrm{EHL}$ made substantial contributions to the conceptualization and methodology, investigation and validation; KBL, CF, JEE, MACL, MVFM and EHL were involved in the formal analysis and agreed to be accountable for all aspects of the work in ensuring that questions related to the accuracy or integrity of any part of the work are appropriately investigated and resolved. They also were involved in drafting the manuscript or revising it critically for important intellectual content. All authors read and approved the final manuscript.

Acknowledgements

Authors want to thank to the Subdirección de Bioinformática at the Instituto Nacional de Medicina Genómica.

Author details

${ }^{1}$ School of Medicine, Autonomous University of the State of Mexico, 50180 Toluca de Lerdo, Mexico.

${ }^{2}$ Computational Genomics Department, National Institute of Genomic Medicine, 14610 Mexico City, Mexico.

${ }^{3}$ Technology Development Department, National Institute of Genomic Medicine, 14610 Mexico City, Mexico.

${ }^{4}$ School of Chemistry, Autonomous University of the State of Mexico, 50120 Toluca de Lerdo, Mexico. ${ }^{5}$ Centro de

Ciencias de la Complejidad, Universidad Nacional Autonoma de Mexico, 04510 Mexico City, Mexico.

\section{References}

1. Gaskin, D.J., Roberts, E.T., Chan, K.S., McCleary, R., Buttorff, C., Delarmente, B.A.: No man is an island: the impact of neighborhood disadvantage on mortality. Int. J. Environ. Res. Public Health 16(7), 1265 (2019)

2. Gavurova, B., Toth, P.: Preventable mortality in regions of slovakia-quantification of regional disparities and investigation of the impact of environmental factors. Int. J. Environ. Res. Public Health 16(8) (2019). doi:10.3390/ijerph16081382

3. Rodríguez-Sanz, M., Gotsens, M., dell?Olmo, M.M., Borrell, C.: Trends in mortality inequalities in an urban area: the influence of immigration. Int. J. Equity Health 18(1), 37 (2019)

4. Ayele, D.G., Zewotir, T.T.: Childhood mortality spatial distribution in ethiopia. J. Appl. Stat. 43(15), 2813-2828 (2016)

5. Burke, M., Heft-Neal, S., Bendavid, E.: Sources of variation in under-5 mortality across sub-saharan africa: a spatial analysis. Lancet Public Health 4(12), 936-945 (2016)

6. Tlou, B., Sartorius, B., Tanser, F.: Space-time patterns in maternal and mother mortality in a rural south african population with high hiv prevalence (2000-2014): results from a population-based cohort. BMC Public Health 17(1), 543 (2017)

7. Montez, J.K., Zajacova, A., Hayward, M.D.: Explaining inequalities in women's mortality between us states. SSM Popul. Health 2, 561-571 (2016)

8. Shin, J., Cho, K., Choi, Y., Lee, S., Park, E.-C., Jang, S.-I.: Combined effect of individual and neighborhood socioeconomic status on mortality in patients with newly diagnosed dyslipidemia: A nationwide korean cohort study from 2002 to 2013. Nutr. Metab. Cardiovasc. Dis. 26(3), 207-215 (2016)

9. Kindig, D.A., Cheng, E.R.: Even as mortality fell in most us counties, female mortality nonetheless rose in 42.8 percent of counties from 1992 to 2006. Health Aff. 32(3), 451-458 (2013)

10. Hazlehurst, M., Nurius, P., Hajat, A.: Individual and neighborhood stressors, air pollution and cardiovascular disease. Int. J. Environ. Res. Public Health 15(3), 472 (2018)

11. Vaughan, A.S., Kramer, M.R., Waller, L.A., Schieb, L.J., Greer, S., Casper, M.: Comparing methods of measuring geographic patterns in temporal trends: an application to county-level heart disease mortality in the united states, 1973 to 2010. Ann. Epidemiol. 25(5), 329-335 (2015)

12. Bethea, T.N., Palmer, J.R., Rosenberg, L., Cozier, Y.C.: Neighborhood socioeconomic status in relation to all-cause, cancer, and cardiovascular mortality in the black women's health study. Ethn. Dis. 26(2), 157 (2016)

13. Dwyer-Lindgren, L., Stubbs, R.W., Bertozzi-Villa, A., Morozoff, C., Callender, C., Finegold, S.B., Shirude, S., Flaxman, A.D., Laurent, A., Kern, E., et al.: Variation in life expectancy and mortality by cause among neighbourhoods in king county, wa, usa, 1990-2014: a census tract-level analysis for the global burden of disease study 2015. Lancet Public Health 2(9), 400-410 (2017)

14. Manda, S.O., Abdelatif, N.: Smoothed temporal atlases of age-gender all-cause mortality in south africa. Int. J. Environ. Res. Public Health 14(9), 1072 (2017)

15. Vaughan, A.S., Schieb, L., Quick, H., Kramer, M.R., Casper, M.: Before the here and now: What we can learn from variation in spatiotemporal patterns of changing heart disease mortality by age group, time period, and birth cohort. Soc. Sci. Med. 217, 97-105 (2018)

16. Parise, C.A., Caggiano, V.: Regional variation in disparities in breast cancer specific mortality due to race/ethnicity, socioeconomic status, and urbanization. J. Racial Ethn. Health Disparities 4(4), 706-717 (2017) 
17. Dwyer-Lindgren, L., Bertozzi-Villa, A., Stubbs, R.W., Morozoff, C., Kutz, M.J., Huynh, C., Barber, R.M., Shackelford, K.A., Mackenbach, J.P., Van Lenthe, F.J., et al.: Us county-level trends in mortality rates for major causes of death, 1980-2014. JAMA 316(22), 2385-2401 (2016)

18. Cho, K.H., Lee, S.G., Nam, C.M., Lee, E.J., Jang, S.-Y., Lee, S.-H., Park, E.-C.: Disparities in socioeconomic status and neighborhood characteristics affect all-cause mortality in patients with newly diagnosed hypertension in korea: a nationwide cohort study, 2002-2013. Int. J. Equity Health 15(1), 3 (2016)

19. Cho, K.H., Nam, C.M., Lee, E.J., Choi, Y., Yoo, K.-B., Lee, S.-H., Park, E.-C.: Effects of individual and neighborhood socioeconomic status on the risk of all-cause mortality in chronic obstructive pulmonary disease: a nationwide population-based cohort study, 2002-2013. Respir. Med. 114, 9-17 (2016)

20. Li, C., Hu, S., Yu, C.: All-cause and cancer mortality trends in macheng, china (1984-2013): An age-period-cohort analysis. Int. J. Environ. Res. Public Health 15(10), 2068 (2018)

21. Ford, M.M., Desai, P.S., Maduro, G., Laraque, F.: Neighborhood inequalities in hepatitis c mortality: spatial and temporal patterns and associated factors. J. Urban Health 94(5), 746-755 (2017)

22. Sifuna, P., Otieno, L., Andagalu, B., Oyieko, J., Ogutu, B., Singoei, V., Owuoth, J., Ogwang, S., Cowden, J., Otieno, W.: A spatiotemporal analysis of hiv-associated mortality in rural western kenya 2011-2015. J. Acquir. Immune Defic. Syndr. 78(5), 483 (2018)

23. Roth, G.A., Abate, D., Abate, K.H., Abay, S.M., Abbafati, C., Abbasi, N., Abbastabar, H., Abd-Allah, F., Abdela, J., Abdelalim, A., et al.: Global, regional, and national age-sex-specific mortality for 282 causes of death in 195 countries and territories, 1980-2017: a systematic analysis for the global burden of disease study 2017. The Lancet 392(10159), 1736-1788 (2018)

24. Rawla, P., Sunkara, T., Gaduputi, V.: Epidemiology of pancreatic cancer: Global trends, etiology and risk factors. World J. Oncol. 10(1), 10 (2019)

25. Dwyer-Lindgren, L., Bertozzi-Villa, A., Stubbs, R.W., Morozoff, C., Mackenbach, J.P., van Lenthe, F.J., Mokdad, A.H., Murray, C.J.: Inequalities in life expectancy among us counties, 1980 to 2014: temporal trends and key drivers. JAMA Intern. Med. 177(7), 1003-1011 (2017)

26. Gouveia, N., Junger, W.L., Romieu, I., Cifuentes, L.A., de Leon, A.P., Vera, J., Strappa, V., Hurtado-Díaz, M., Miranda-Soberanis, V., Rojas-Bracho, L., et al.: Effects of air pollution on infant and children respiratory mortality in four large latin-american cities. Environ. Pollut. 232, 385-391 (2018)

27. Salto-Quintana, J.N., Rivera-Alfaro, G., Sánchez-Ramos, E.L., Gómez-Gómez, A., Noyola, D.E.: Post-pandemic influenza-associated mortality in mexico. Pathog. Glob. Health, 1-8 (2019)

28. Wang, N., Mengersen, K., Tong, S., Kimlin, M.G., Zhou, M., Wang, L., Hu, W.: Lung cancer mortality in china: Spatial and temporal trends among subpopulations. Available at SSRN 3315845 (2019)

29. Wändell, P., Carlsson, A.C., Gasevic, D., Sundquist, J., Sundquist, K.: Neighbourhood socio-economic status and all-cause mortality in adults with atrial fibrillation: a cohort study of patients treated in primary care in sweden. Int. J. Cardiol. 202, 776-781 (2016)

30. Yang, W., Olson, D.R., Shaman, J.: Forecasting influenza outbreaks in boroughs and neighborhoods of new york city. PLOS Comput. Biol. 12(11), 1005201 (2016)

31. Ryvicker, M., Sridharan, S.: Neighborhood environment and disparities in health care access among urban medicare beneficiaries with diabetes: A retrospective cohort study. NQUIRY J. Health Car 55, 0046958018771414 (2018)

32. Goovaerts, P.: Geostatistical analysis of disease data: estimation of cancer mortality risk from empirical frequencies using poisson kriging. Int. J. Health Geogr. 4(1), 31 (2005)

33. Lin, W.-C., Lin, Y.-P., Wang, Y.-C., Chang, T.-K., Chiang, L.-C.: Assessing and mapping spatial associations among oral cancer mortality rates, concentrations of heavy metals in soil, and land use types based on multiple scale data. Int. J. Environ. Res. Public Health 11(2), 2148-2168 (2014)

34. Tadesse, S., Enqueselassie, F., Gebreyesus, S.H.: Estimating the spatial risk of tuberculosis distribution in gurage zone, southern ethiopia: a geostatistical kriging approach. BMC Public Health 18(1), 783 (2018)

35. Chien, L.-C., Yu, H.-L., Schootman, M.: Efficient mapping and geographic disparities in breast cancer mortality at the county-level by race and age in the us. Spatial Spatio-temporal Epidemiol. 5, 27-37 (2013)

36. Ramos, G.C.D., et al.: Real estate industry as an urban growth machine: A review of the political economy and political ecology of urban space production in mexico city. Sustainability 11(7), 1-24 (2019)

37. Aburto, J.M., Riffe, T., Canudas-Romo, V.: Trends in avoidable mortality over the life course in mexico, 1990-2015: a cross-sectional demographic analysis. BMJ Open 8(7), 022350 (2018)

38. Benita, F.: Social backwardness in mexico city metropolitan area. Soc. Indic. Res. 126(1), 141-160 (2016)

39. datamx Colonias México. http://datamx.io/dataset/colonias-mexico. Accessed: 2019-02-01

40. Bivand, R., Rundel, C.: Rgeos: Interface to Geometry Engine - Open Source ('GEOS'). (2018). R package version 0.4-2. https://CRAN.R-project.org/package=rgeos

41. Secretaría de Salud de la Ciudad de México. http://data.salud.cdmx.gob.mx/portal/. Accessed: 2019-02-01

42. Secretaría de Salud de la Ciudad de México: Mortalidad. http://data.salud.cdmx.gob.mx/portal/index.php/informacion-en-salud/103-informacion-salud/354-mortalidad Accessed: 2019-02-01

43. Instituto Nacional de Estadística y Geografía. https://www.inegi.org.mx/. Accessed: 2019-02-15

44. Oliver, M., Webster, R.: A tutorial guide to geostatistics: Computing and modelling variograms and kriging. Catena 113, 56-69 (2014)

45. Pebesma, E.J.: Multivariable geostatistics in S: the gstat package. Computers \& Geosciences 30, 683-691 (2004)

46. Gräler, B., Pebesma, E., Heuvelink, G.: Spatio-temporal interpolation using gstat. The R Journal 8, 204-218 (2016)

47. Pinheiro, J., Bates, D.: Mixed-effects Models in S and S-PLUS. Springer, ??? (2006)

48. Di Rienzo, J., Casanoves, F., Balzarini, M., Gonzalez, L., Tablada, M., Robledo, C.: InfoStat. Universidad Nacional de Córdoba (2011)

49. R Core Team: R: A Language and Environment for Statistical Computing. R Foundation for Statistical 
Computing, Vienna, Austria (2018). R Foundation for Statistical Computing. https://www.R-project.org/

50. Pinheiro, J., Bates, D., DebRoy, S., Sarkar, D., R Core Team: nlme: Linear and Nonlinear Mixed Effects Models. (2018). R package version 3.1-137. https://CRAN.R-project.org/package=nlme

51. Programa Delegacional de Desarrollo en Cuauhtémoc 2016-2018.

http://www.data.seduvi.cdmx.gob.mx/portal/index.php/programas-de-desarrollo/programas-delegacionales. Accessed: 2019-04-01

52. Secretariado Ejecutivo del Sistema Nacional de Seguridad Pública. https://www.gob.mx/sesnsp. Accessed: 2019-04-01

Figures

Figure 1 Mexico City study area. A) Borough level description of the 16 cases (in numbers) corresponding to: 1. Álvaro Obregón, 2. Azcapotzalco, 3. Benito Juárez, 4. Coyoacán, 5. Cuajimalpa de Morelos, 6. Cuauhtémoc, 7. Gustavo A. Madero, 8. Iztacalco, 9. Iztapalapa, 10. La Magdalena Contreras, 11. Miguel Hidalgo, 12. Milpa Alta, 13. Tláhuac, 14. Tlalpan, 15.

Venustiano Carranza and 16. Xochimilco . B) Neighbourhood level description. Blue lines describe borough limits whereas white lines at neighbourhood areas (in gray). Red dots depict borough calculated centroids. In both panels the scale bar and north arrow are also included. Notice that some boroughs have a dense neighbourhood description in comparison.

Figure 2 Age-specific mortality rates in Mexico city. Each row corresponds to a particular age-specific mortality rate, i.e., Global, Post-productive, Productive, School, Pre-school and Infant, whereas columns stand for selected years 2000, 2005, 2010 and 2015 from the total period 2000 to 2016. Boroughs are colour coded according to the corresponding mortality rate, which make them only comparable by row. On the contrary, notice the different mortality rate ranges (colour bars) depending in the age-specific group of analysis.

Figure 3 Mortality rates in Mexico City at borough level. Each panel corresponds to an age-specific mortality rate: i) Infant, ii) Pre-school, iii) School, iv) Productive, v) Post-productive and vi) Global. All panels include complete year records from 2000 up to 2016, where age-specific mortality rates are presented by points, boroughs in color and the dashed lines try to mimic the time-evolution patterns. Interestingly, as the years pass, the mortality rate time evolution seems to diminish for Infant and Post-productive groups, whereas the Global and Productive age-specific counterpart tends to increase. In all cases, data were obtained from the Secretariat of Health in Mexico City (Secretaría de Salud de la Ciudad de México, SEDESA) [41].

Figure 4 Three levels of granularity for global mortality rate in Mexico city. A) First global mortality rate original data description at borough level. B) Second global mortality rate kriged data description at neighbourhood level. C) Zoom in at a second description level for Cuauhtémoc borough. Interestingly, this is the borough with the highest mortality rate no matter the selected year (2000, 2005, 2010 or 2015), according to panel A and B (central map in red). However, the mortality rate is not homogeneous nor constant at neighbourhood level, as depicted by the kriged values presented in panel $\mathrm{C}$ for the different years.

Figure 5 Mortality rate posterior tests. Fisher's Least Significant Difference (LSD) performed over the estimated mean mortality rate of the fixed effect terms: A) Age-specific mortality, B) Borough level, C) Age-specific $\times$ Borough interaction. In all cases, capital letters stand for Fisher's LSD groups, where bars that share at least a single letter, are not statistically different after Bonferroni multiple test correction $(p>0.05)$. Results are presented as ordered mean \pm standard error estimation according to model description of Equations (1-4).

Tables

Additional Files

Additional file 1 - Variogram modeling for age-specific mortality rates. 
Figure 6 Posterior test results at Cuauhtémoc borough. A) Neighbourhood level description where, numbers are order from the highest to the lowest model estimated mortality mean respectively: 1. Tabacalera, 2. Centro, 3. Juárez, 4. Doctores, 5. Buenavista, 6. Guerrero, 7. San Rafael, 8. Roma Norte, 9. Obrera, 10. Cuauhtémoc, 11. Santa María la Ribera, 12. Tránsito, 13. Centro Urbano Benito Juárez, 14. Esperanza, 15. Unidad Hab. Nonoalco Tlatelolco, 16. Morelos, 17. Buenos Aires, 18. Atlampa, 19. Vista Alegre, 20. Algarín, 21. Paulino Navarro, 22. Roma Sur, 23. Ex-hipódromo de Peralvillo, 24. San Simón Tolnáhuac, 25. Santa María Insurgentes, 26. Hipódromo, 27. Maza, 28. Ampl. Asturias, 29. Felipe Pescador, 30. Condesa, 31. Asturias, 32. Peralvillo, 33. Valle Gómez and 34. Hipódromo de la Condesa. B) Neighbourhoods are filled according to the Fisher's Least Significant Difference (LSD) group obtained over at this level. C) Age-specific Fisher's LSD results at Cuauhtémoc borough. D) Fisher's LSD neighbourhood contribution. In all cases, capital letters stand for Fisher's LSD groups, where bars that share at least a single letter, are not statistically different after Bonferroni multiple test correction $(p>0.05)$. Results are presented as ordered mean \pm standard error estimation according to model description of Equation (1-4).

Table 1 Analysis of the Variance at borough and neighbourhood level. Type III sum of squares was used to assess the model defined in Equations (1-4), where, $\mu$, is the global mean; $\alpha_{i}$ the age-specific mortality term; $\beta_{j}$ the borough or neighbourhood term; $t, t^{2}$ and $t^{3}$ the third order time polynomial; and the double and triple interactions accordingly. This definitions were used at both spatial levels, i. e., borough and neighbourhood. In addition, fixed effects, back-step model selection was carried out from the maximal to the current model at neighbourhood level. Empty cell correspond to discarded terms.

\begin{tabular}{|c|c|c|c|c|c|c|}
\hline \multirow[b]{2}{*}{ Model Term } & \multicolumn{3}{|c|}{ Borough level } & \multicolumn{3}{|c|}{ Neighbourhood level } \\
\hline & $\begin{array}{l}\text { Degrees of } \\
\text { freedom }\end{array}$ & F-value & p-value & $\begin{array}{l}\text { Degrees of } \\
\text { freedom }\end{array}$ & F-value & p-value \\
\hline$\mu$ & 1 & 17634.6381 & $<0.0001$ & 1 & 281297.3649 & $<0.0001$ \\
\hline$\alpha_{i}$ & 5 & 6981.1137 & $<0.0001$ & 5 & 6929.1962 & $<0.0001$ \\
\hline$\beta_{j}$ & 15 & 8.3045 & $<0.0001$ & 33 & 6.5863 & $<0.0001$ \\
\hline$t$ & 1 & 7.9808 & 0.0048 & 1 & 714.5003 & $<0.0001$ \\
\hline$t^{2}$ & 1 & 34.1072 & $<0.0001$ & 1 & 248.0783 & $<0.0001$ \\
\hline$t^{3}$ & 1 & 33.6751 & $<0.0001$ & 1 & 430.7367 & $<0.0001$ \\
\hline$\alpha_{i} \times \beta_{j}$ & 75 & 20.0209 & $<0.0001$ & 165 & 5.6587 & $<0.0001$ \\
\hline$\beta_{j} \times t$ & 15 & 2.9735 & 0.0001 & 33 & 9.0428 & $<0.0001$ \\
\hline$\beta_{j} \times t^{2}$ & 15 & 2.4779 & 0.0013 & 33 & 2.2043 & $<0.0001$ \\
\hline$\beta_{j} \times t^{3}$ & 15 & 1.9233 & 0.0177 & & & \\
\hline$\alpha_{i} \times t$ & 5 & 4.172 & 0.0009 & 5 & 69.3982 & $<0.0001$ \\
\hline$\alpha_{i} \times t^{2}$ & 5 & 7.2432 & $<0.0001$ & 5 & 88.1181 & $<0.0001$ \\
\hline$\alpha_{i} \times t^{3}$ & 5 & 7.4925 & $<0.0001$ & 5 & 89.7661 & $<0.0001$ \\
\hline$\alpha_{i} \times \beta_{j} \times t$ & 75 & 1.7383 & 0.0001 & 165 & 4.9837 & $<0.0001$ \\
\hline$\alpha_{i} \times \beta_{j} \times t^{2}$ & 75 & 1.3908 & 0.0175 & & & \\
\hline$\alpha_{i} \times \beta_{j} \times t^{3}$ & 75 & 1.1254 & 0.2224 & & & \\
\hline
\end{tabular}


Figures

A

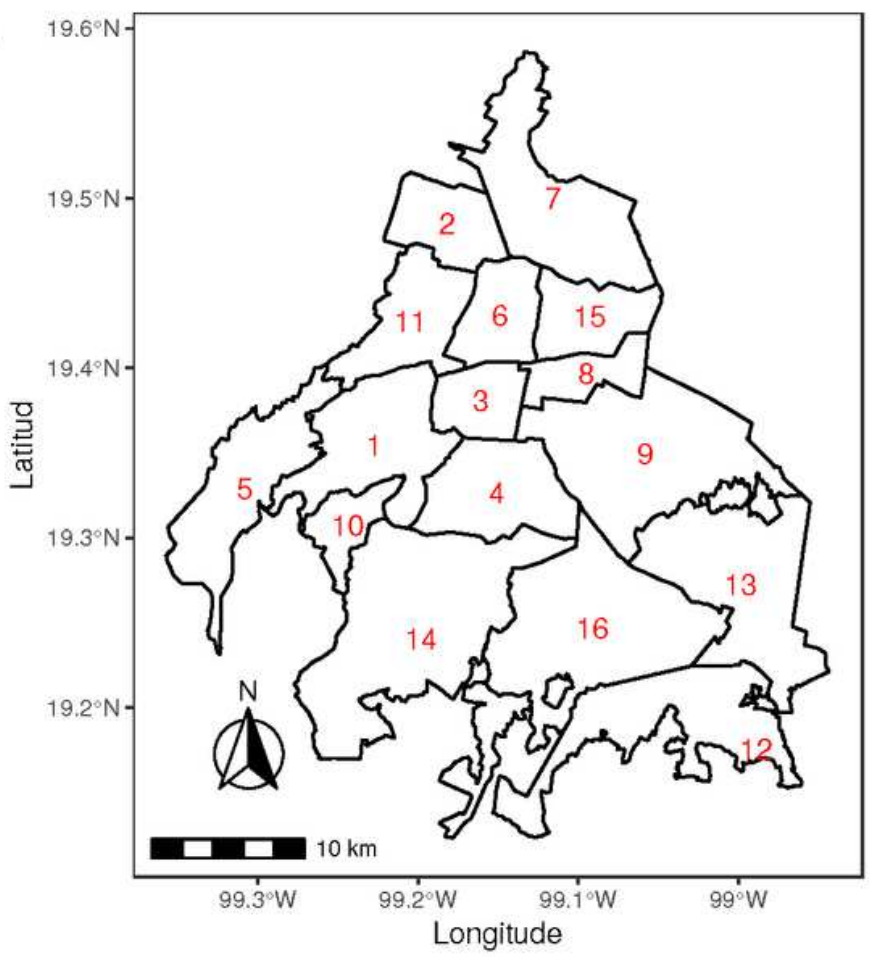

B

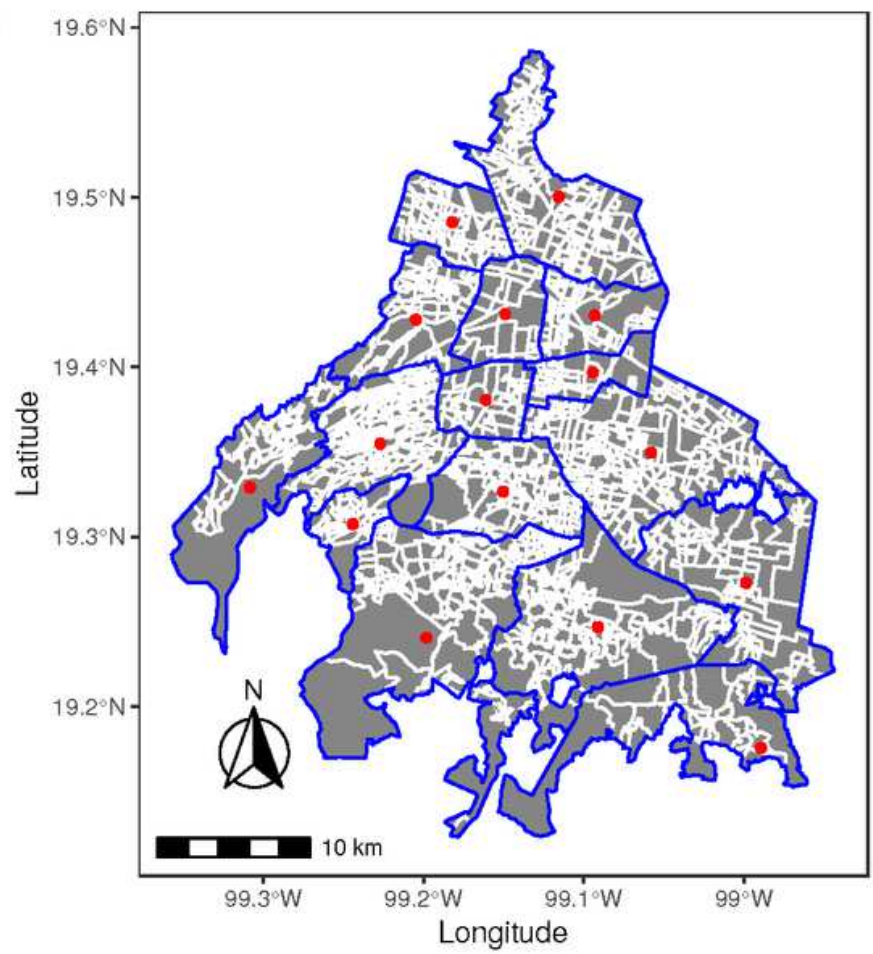

Figure 2

Mexico City study area. A) Borough level description of the 16 cases (in numbers) corresponding to: 1 . 'Alvaro Obreg'on, 2. Azcapotzalco, 3. Benito Ju'arez, 4. Coyoac'an, 5. Cuajimalpa de Morelos, 6. Cuauht 'emoc, 7. Gustavo A. Madero, 8. Iztacalco, 9. Iztapalapa, 10. La Magdalena Contreras, 11. Miguel Hidalgo, 12. Milpa Alta, 13. Tl'ahuac, 14. Tlalpan, 15. Venustiano Carranza and 16. Xochimilco . B) Neighbourhood level description. Blue lines describe borough limits whereas white lines at neighbourhood areas (in gray). Red dots depict borough calculated centroids. In both panels the scale bar and north arrow are also included. Notice that some boroughs have a dense neighbourhood description in comparison. 

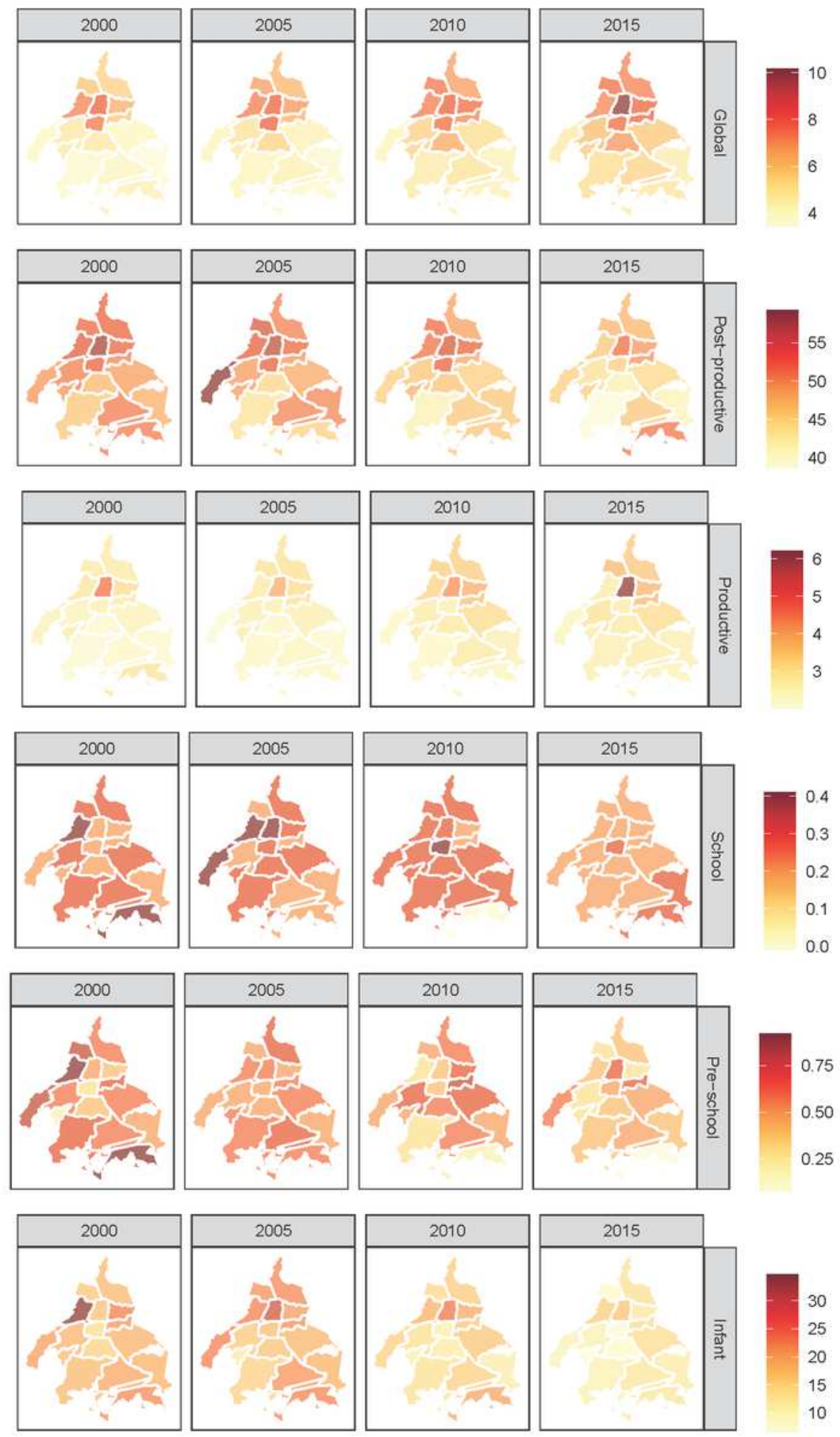

\section{0 \\ 25 \\ 20 \\ 10}

\section{Figure 3}

Age-specific mortality rates in Mexico city. Each row corresponds to a particular age-specific mortality rate, i.e., Global, Post-productive, Productive, School, Pre-school and Infant, whereas columns stand for selected years 2000, 2005, 2010 and 2015 from the total period 2000 to 2016. Boroughs are colour coded according to the corresponding mortality rate, which make them only comparable by row. On the contrary, notice the different mortality rate ranges (colour bars) depending in the age-specific group of analysis. 


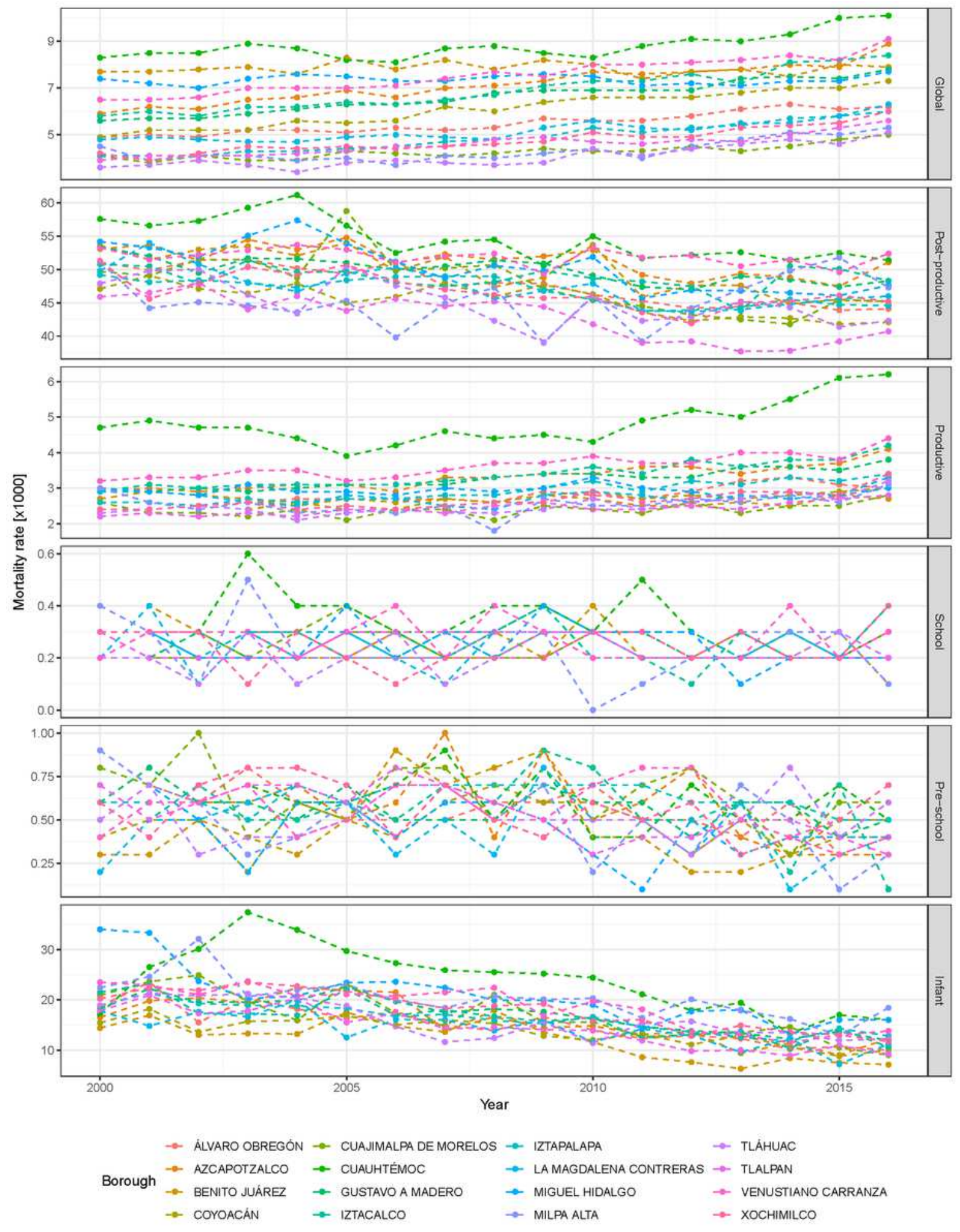

\section{Figure 6}

Mortality rates in Mexico City at borough level. Each panel corresponds to an age-specific mortality rate: i) Infant, ii) Pre-school, iii) School, iv) Productive, v) Post-productive and vi) Global. All panels include complete year records from 2000 up to 2016, where age-specific mortality rates are presented by points, boroughs in color and the dashed lines try to mimic the time-evolution patterns. Interestingly, as the years pass, the mortality rate time evolution seems to diminish for Infant and Post-productive groups, whereas 
the Global and Productive age-specific counterpart tends to increase. In all cases, data were obtained from the Secretariat of Health in Mexico City (Secretar'ıa de Salud de la Ciudad de M'exico, SEDESA) [41].
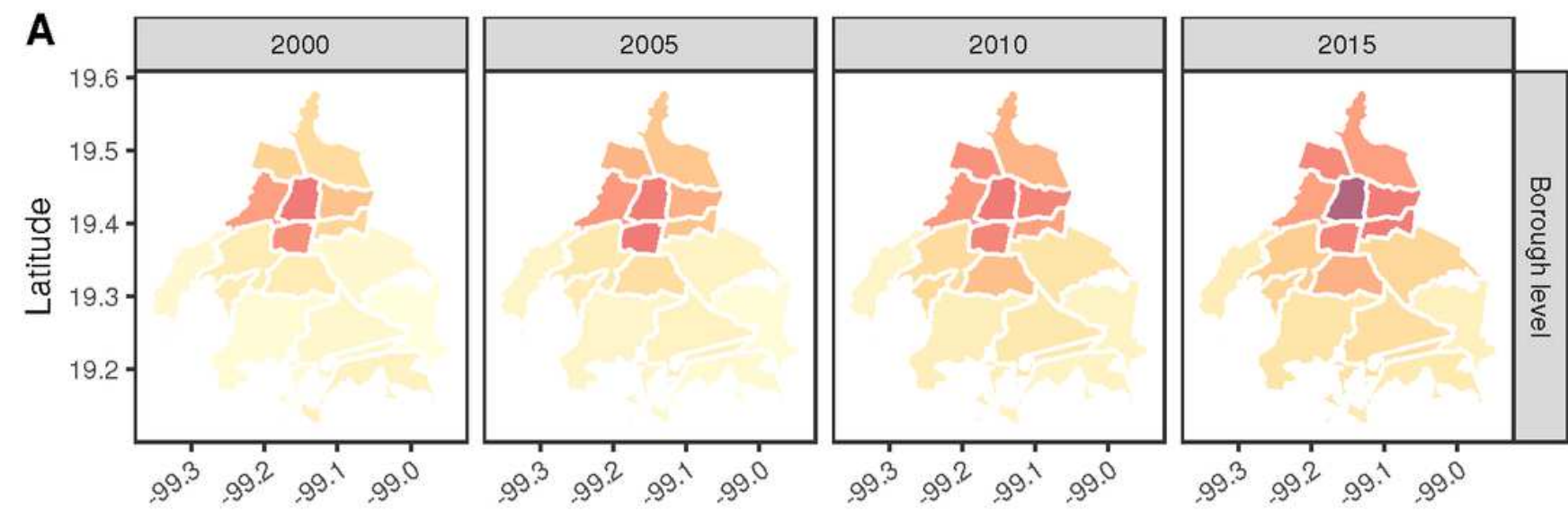

Rate

Longitude
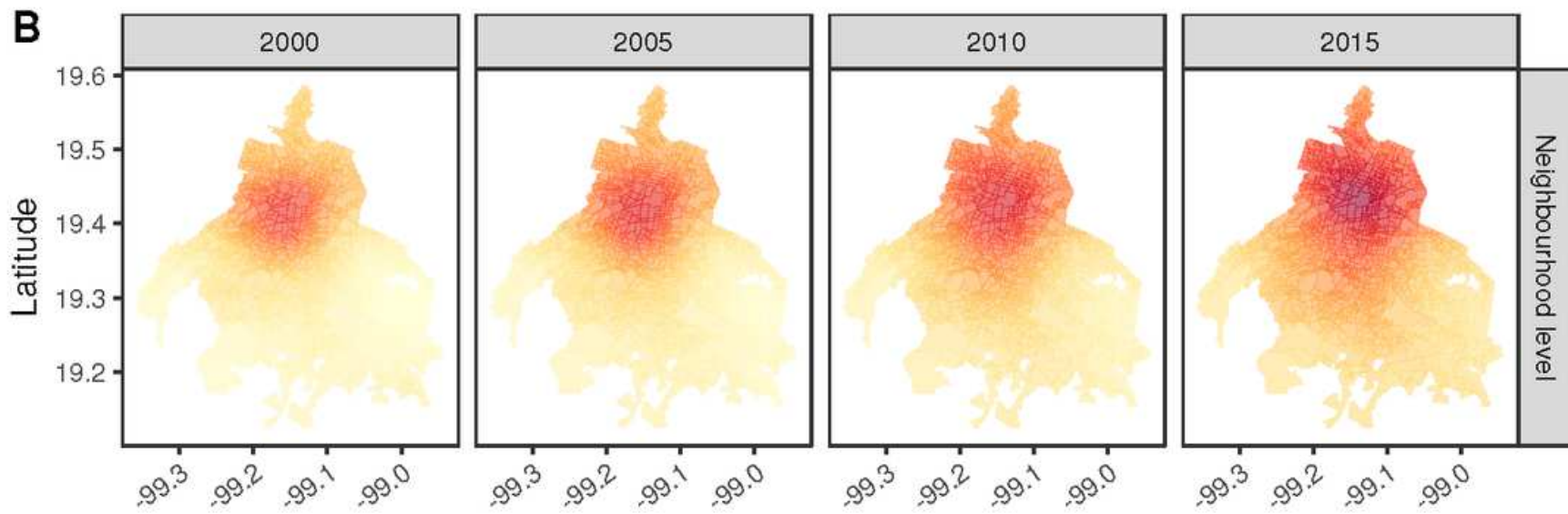

Rate
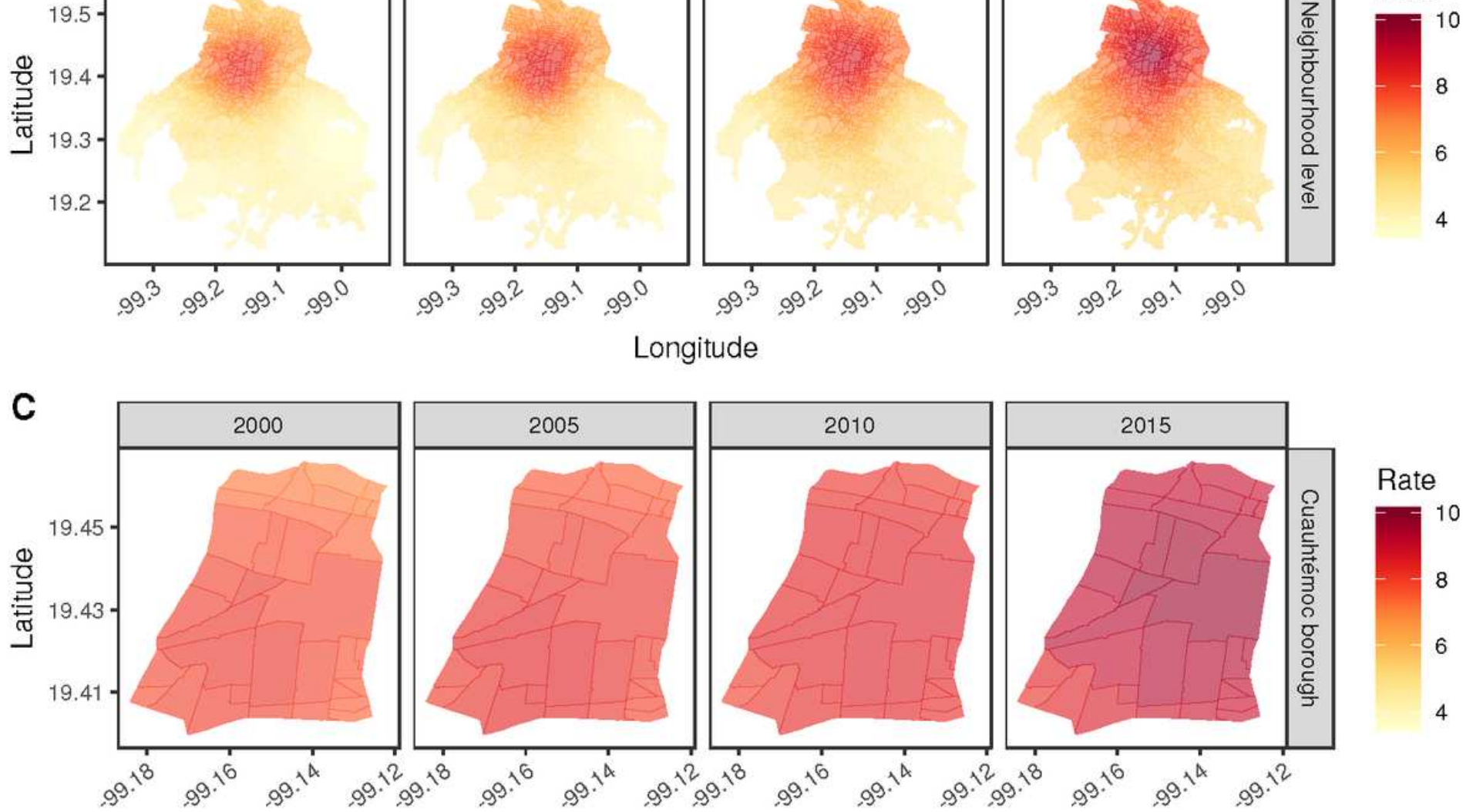

$.99^{16} \cdot 99^{10}$

\section{Longitude}

\section{Figure 8}

Three levels of granularity for global mortality rate in Mexico city. A) First global mortality rate original data description at borough level. B) Second global mortality rate kriged data description at neighbourhood level. C) Zoom in at a second description level for Cuauht'emoc borough. Interestingly, this is the borough with the highest mortality rate no matter the selected year $(2000,2005,2010$ or 2015$)$, 
according to panel $A$ and $B$ (central map in red). However, the mortality rate is not homogeneous nor constant at neighbourhood level, as depicted by the kriged values presented in panel $\mathrm{C}$ for the different years.
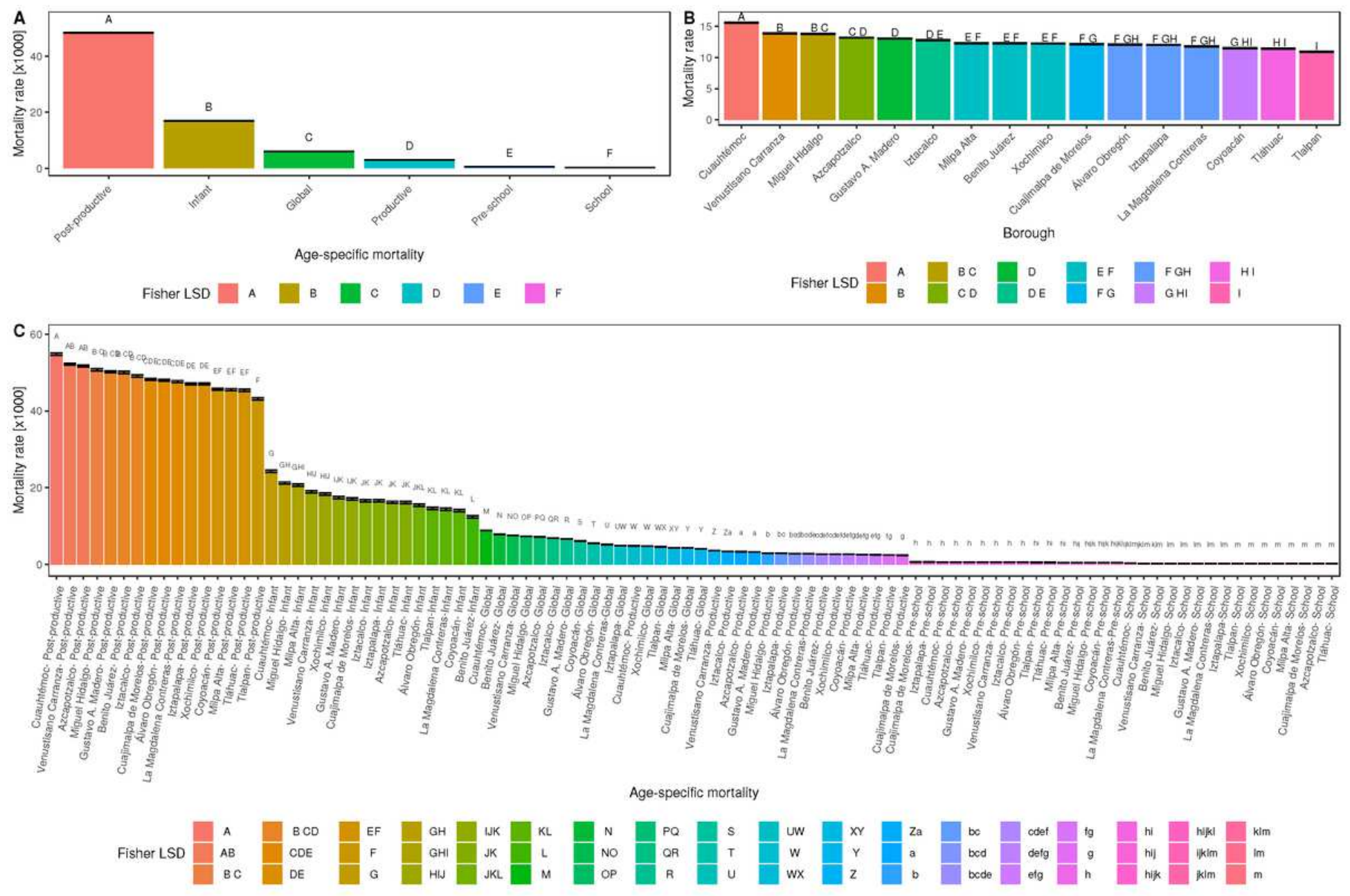

\section{Figure 10}

Mortality rate posterior tests. Fisher's Least Significant Difference (LSD) performed over the estimated mean mortality rate of the fixed effect terms: A) Age-specific mortality, B) Borough level, C) Age-specific $\times$ Borough interaction. In all cases, capital letters stand for Fisher's LSD groups, where bars that share at least a single letter, are not statistically different after Bonferroni multiple test correction $(p>0.05)$. Results are presented as ordered mean \pm standard error estimation according to model description of Equations (1-4). 
A
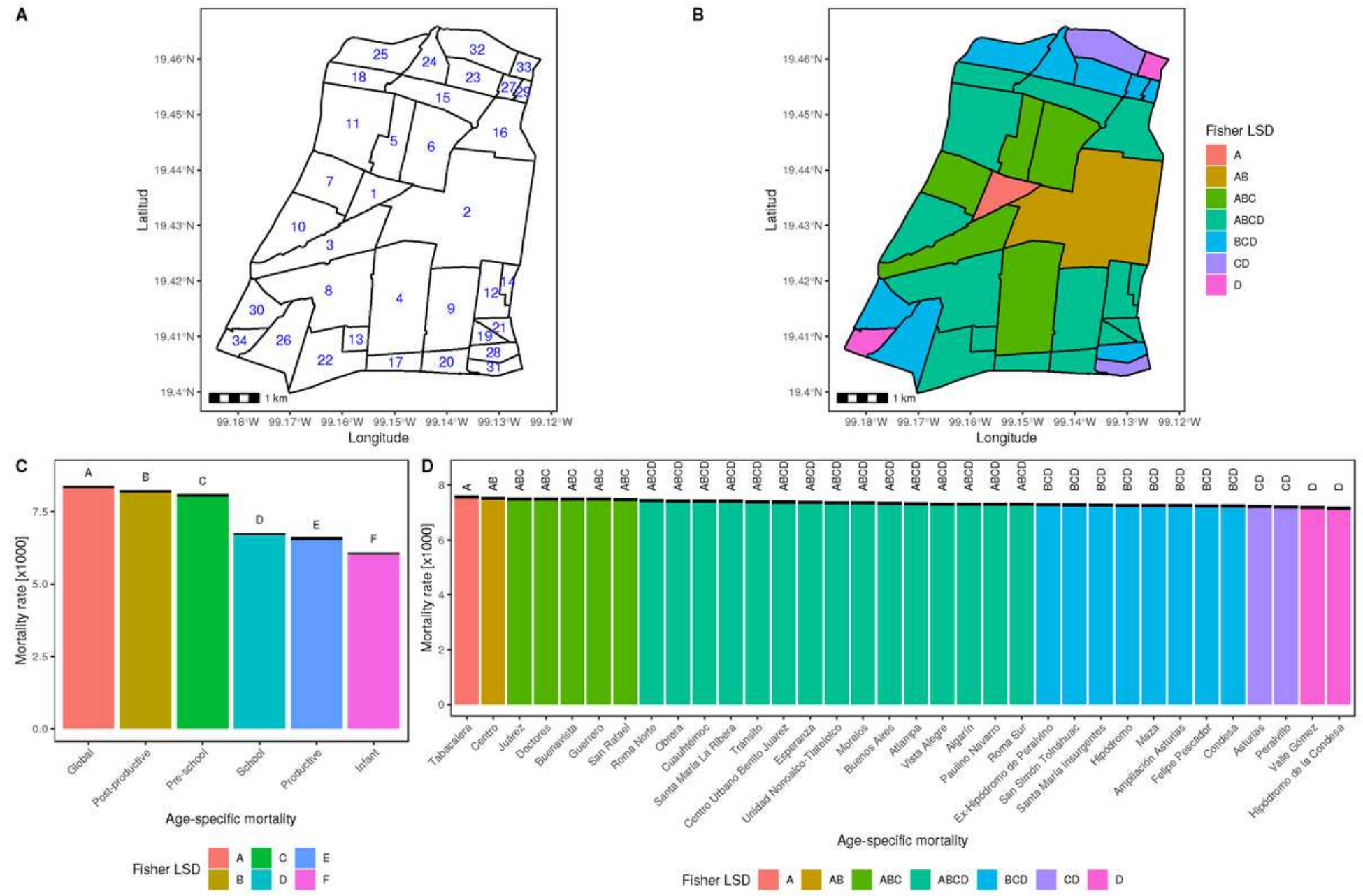

Figure 11

Posterior test results at Cuauht'emoc borough. A) Neighbourhood level description where, numbers are order from the highest to the lowest model estimated mortality mean respectively: 1. Tabacalera, 2 . Centro, 3. Ju'arez, 4. Doctores, 5. Buenavista, 6. Guerrero, 7. San Rafael, 8. Roma Norte, 9. Obrera, 10. Cuauht'emoc, 11. Santa Mar'ıa la Ribera, 12. Tr'ansito, 13. Centro Urbano Benito Ju'arez, 14. Esperanza, 15. Unidad Hab. Nonoalco Tlatelolco, 16. Morelos, 17. Buenos Aires, 18. Atlampa, 19. Vista Alegre, 20. Algar'ın, 21. Paulino Navarro, 22. Roma Sur, 23. Ex-hip'odromo de Peralvillo, 24. San Sim'on Toln'ahuac, 25. Santa Mar'ıa Insurgentes, 26. Hip'odromo, 27. Maza, 28. Ampl. Asturias, 29. Felipe Pescador, 30. Condesa, 31. Asturias, 32. Peralvillo, 33. Valle G'omez and 34. Hip'odromo de la Condesa. B) Neighbourhoods are filled according to the Fisher's Least Significant Difference (LSD) group obtained over at this level. C) Age-specific Fisher's LSD results at Cuauht'emoc borough. D) Fisher's LSD neighbourhood contribution. In all cases, capital letters stand for Fisher's LSD groups, where bars that share at least a single letter, are not statistically different after Bonferroni multiple test correction $(p>$ 0.05 ). Results are presented as ordered mean \pm standard error estimation according to model description of Equation (1-4).

\section{Supplementary Files}


This is a list of supplementary files associated with this preprint. Click to download.

- supplementary.pdf

- supplementary.pdf 